\title{
Low Power Dropout Regulators with Efficiently Controlled Transient Response
}

\author{
Varun Kumar Singhal
}

Tarun Kumar Gupta, PhD

\begin{abstract}
The Low-Dropout voltage regulators are significant part of VLSI design integrated chips and are used to provide steady supply voltage to noise sensitive analog/RF circuit blocks. It belongs to class of linear regulators designed to minimize the saturation of output pass transistor and its drive requirements. A capacitor-less low-dropout regulator with improved performance push-pull power transistor is described in the paper. The proposed LDO is stable over a wide range of load current and implemented in 65-nm CMOS process technology. The simulation illustrates that the regulator is able to convert $V_{\text {IN }}$ of $0.65 \mathrm{~V}-1.5 \mathrm{~V}$ to output voltage of $0.5 \mathrm{~V}$. This LDO achieves power supply rejection of $>40 \mathrm{~dB}$ at $1 \mathrm{kHz}$ operating frequency. It consumes a quiescent current less than $10 \mathrm{uA}$. It is capable of delivering a maximum load current of $70 \mathrm{~mA}$ with a dropout voltage of less than $220 \mathrm{mV}$.
\end{abstract}

\section{Keywords}

Low dropout, regulators, low quiescent current, enhanced, capacitor-less.

\section{INTRODUCTION}

POWER management unit with several integrated regulators is widely used in modern battery-operated portable devices. These power controlling schemes often use a primary switching regulator and several post-regulators [1], [2]. The primary switching regulator converts the high dc voltage level of the battery into a low dc voltage with a high conversion proficiency $(>90 \%)$. The post-regulators also generate several autonomous

power sources for multiple voltage domains. The switching regulator certainly generates voltage ripples over the range of the switching frequency. The switching frequency response of the regulator often lies within a low-frequency band of a few $10-500 \mathrm{kHz}$ to diminish switching power loss. The postregulators should, therefore, be able to provide a good power supply rejection ratio (PSRR) ability to suppress the undesirable low-frequency noise. To promote high power efficiency, abating the influence on load circuits, these postregulators must operate at low voltage and low quiescent current (IQ), achieve a fast transient response with a small output deviation.

Dropout voltage can be reduced by increasing the width of the pass transistor.

As the width of pass transistor is increased, capacitance associated with the pass transistor also increases and thus charging and discharging of the pass capacitance also increases. This results in degradation of the transient response and causes stability issues.

Several techniques were proposed in the past to overcome the slew rate limitation at the gate of pass transistor and improve the transient response. Work in [3] discusses the current efficient buffer and current boosted pass device for low voltage applications consuming quiescent current of $23 \mu \mathrm{A}$ at no load. This LDO would use electrostatic series resistance of the output capacitor to maintain stability. In [4] capacitive feedback frequency recompense scheme was presented to get rid off dependence on effective series-resistance of output capacitor for the purpose of stability. However, reducing the quiescent current in a regulator results in slow transient behaviour of circuit. To meet the obligation of fast regulation and small dropout voltage [5] is proposed achieving a quiescent current of $6 \mathrm{~mA}$ having current efficiency of $94 \%$ and is stabilized by $0.6 \mathrm{Nf}$ on-chip capacitor.

[6] Determine gain enhanced flipped voltage follower having quiescent current of $8 \mu \mathrm{A}$ but it entails minimum load current of $3 \mathrm{~mA}$ to certify stability for CL of $50 \mathrm{pF}$. Hence, it is not proper for low load current applications. To solve slew rate problems, push-pull technique is demonstrated but they also need minimum load current to maintain stability. In [7], it proposed an assistant push-pull output stage to improve slew rate for improved transient performance without requiring any on chip or off-chip capacitor. But this also requires minimum load current of $100 \mu \mathrm{A}$ to maintain stability.

In this paper an enhanced low dropout voltage regulator is presented. This technique makes the circuit fully signal dependent dynamically operated. By excluding the current mirror M8-M9 (fig 1), quiescent current is minimized and thus efficiency of the regulator is increased. This makes use of modest frequency recompense scheme with minor compensation capacitor.

The various section of this paper are described below. The proposed transient enhanced LDO with modified push-pull power transistor is presented in section II. Stability analysis and standard operation is discussed in section III. Section IV defines simulation results and comparison. Section V describes the conclusion part.

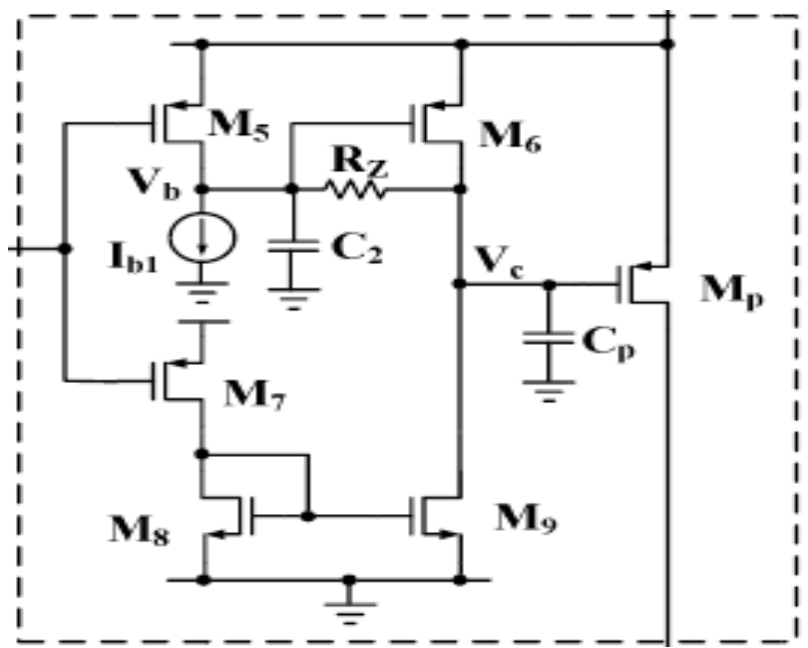

Fig. 1: Conventional push-pull composite power transistor 


\section{PROPOSED PUSH-PULL POWER TRANSISTOR}

For low voltage operating environment and enough loop gain, LDO with multi stage structures are often adopted. With single frequency compensation, class A composite power transistor is proposed in [8], [9]. This structure offers the advantage of higher stability over other designs. Also, transconductance and bandwidth can be adjusted independently. But it suffers from sinking capability problem. To solve this, LDO with push-pull composite power transistor is proposed in [10]. Schematic of modified push-pull composite power transistor is shown in Fig.2. Transistors M7-M9 serves as a signal dependent current source. Thus, bias current of M6 and M9 depends on the voltage level at the gate of power transistor. Sinking capability at node Vc is no longer limited with the signal dependent voltage source.
$G_{m p(p u s h-p u l l)}=\frac{\left(g m 5 R_{Z}+\frac{g m 7}{g m 6}\right)}{\left(1+\frac{C_{P}\left(R_{2}+R_{Z}\right)}{g m 6 R_{2}} s\right)} \frac{\left(1+\frac{g m 7 C_{2}}{g m 5 g m 6} s\right)}{\left(1+\frac{C_{2} R_{Z} R_{2}}{\left(R_{2}+R_{Z}\right)} s\right)} \times g m p$

From the above equation, it is clear that the trans-conductance of push-pull composite power transistor is larger as compared to class-A due to signal reliant on the current source. Transconductance and bandwidth are also self-determining to each other as in class-A push pull circuit. But this structure consumes a sizable amount of quiescent current which is the main issue for low voltage applications.

To overcome this issue, current mirror transistors in pull down network are excluded and the circuit is made fully signal dependent. This circuitry reduces the current consumed by the dropout regulator and hence its efficiency improves.

From the small signal model analysis, frequency dependent trans-conductance can be obtained as,

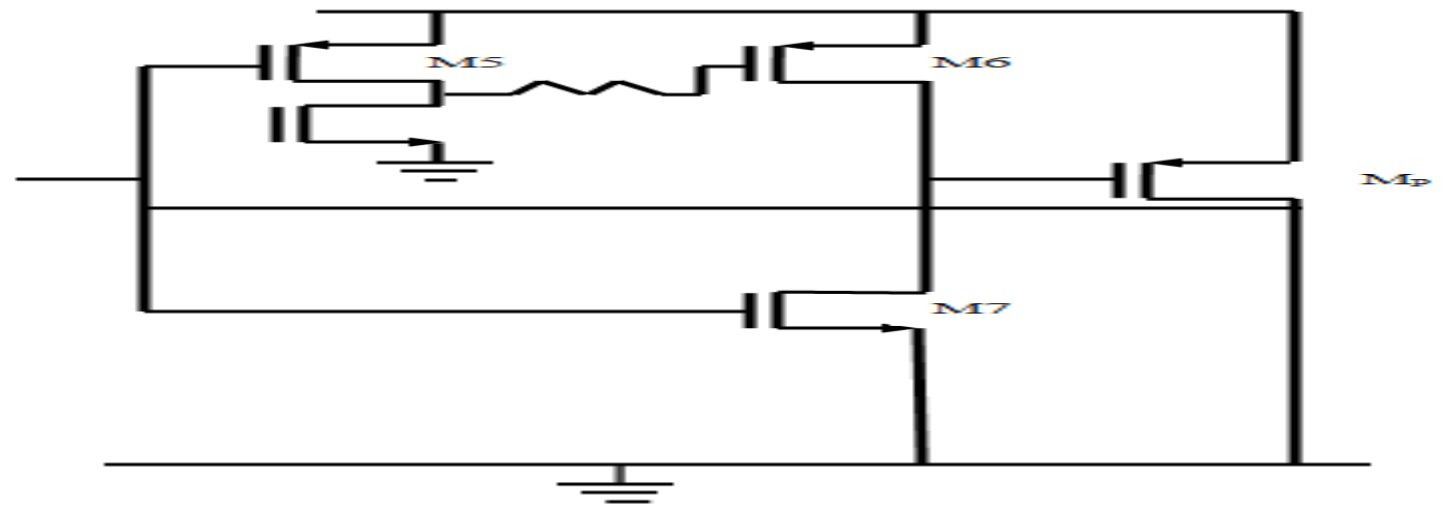

Fig. 2 Proposed push-pull composite power transistor

\section{PROPOSED LDO REGULATOR}

The schematic circuitry of the proposed LDO with modified push-pull composite power transistor is presented in Fig. 3. The two transistors in pull up network form the differential pair where as the other two transistors in pull down form the current mirror and $\mathrm{Mb} 1$ serves as the current source for error amplifier. The proposed push-pull composite power transistor is formed by a low-voltage embedded gain stage and a power transistor MP. Miller compensation is utilized with miller capacitance $\mathrm{Cm}$ and $\mathrm{CP}$ is the parasitic capacitance at the gate of pass transistor MP. The two resistors form the resistive voltage divider feedback network.

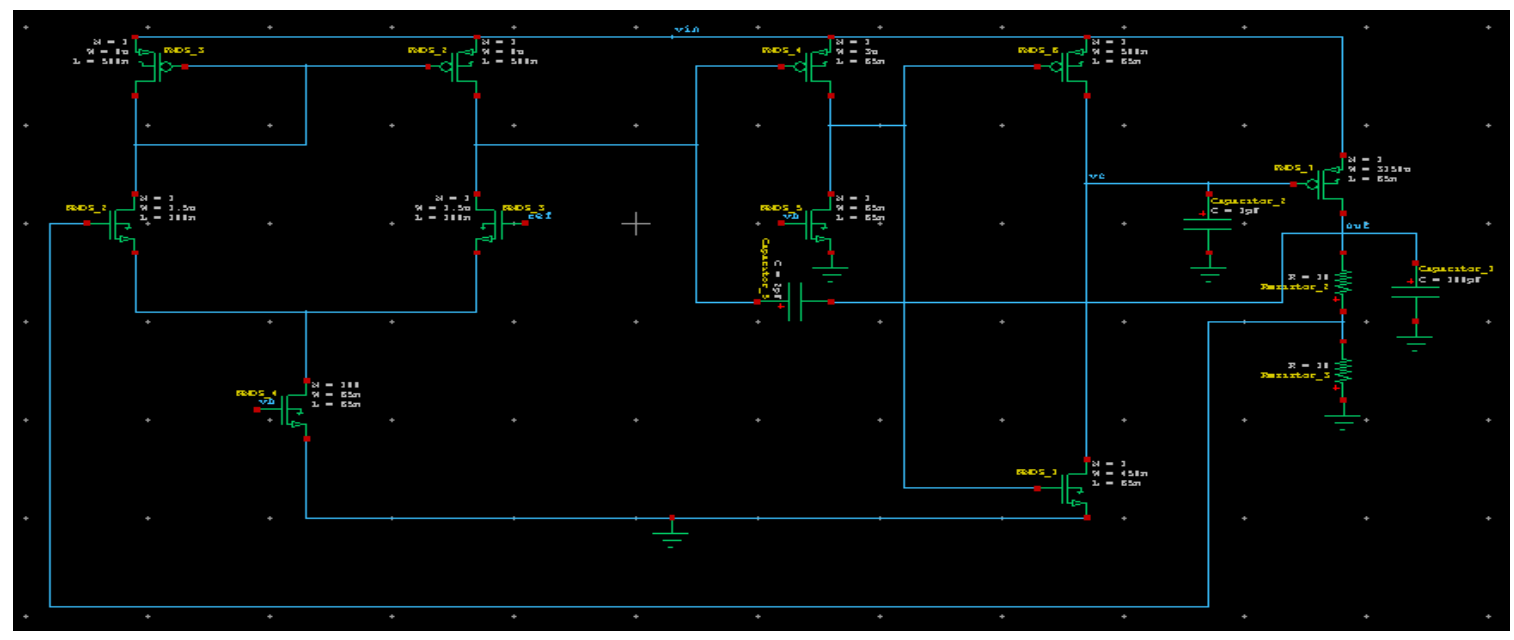

Fig 3. Proposed Low Dropout Regulator

\section{SIMULATION RESULTS}

The proposed Low dropout regulator is simulated in cadence virtuoso in $65 \mathrm{~nm}$ CMOS process technology and the corresponding results are obtained. The simulation waveforms of the proposed LDO work are shown in the fig 4-7. The corresponding results are shown in the Table 1: 


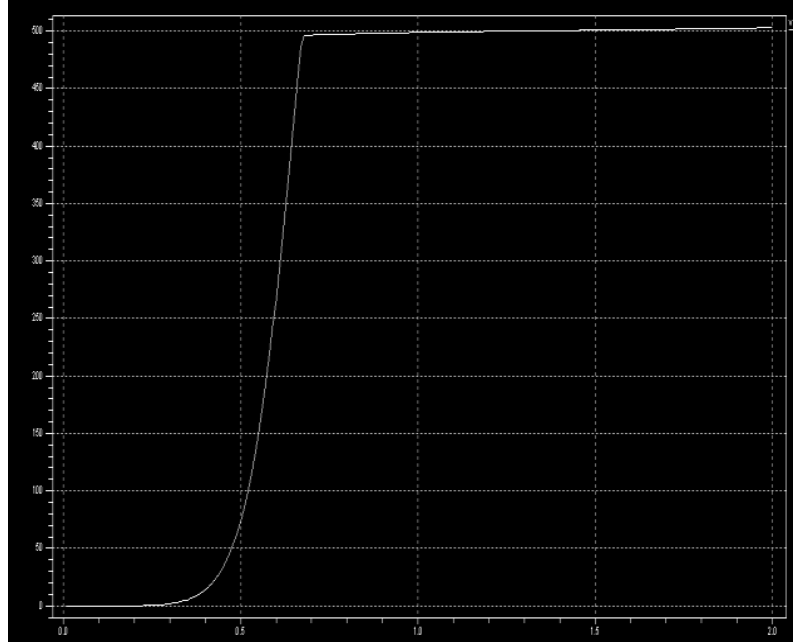

Fig.4 DC characteristics of proposed regulator

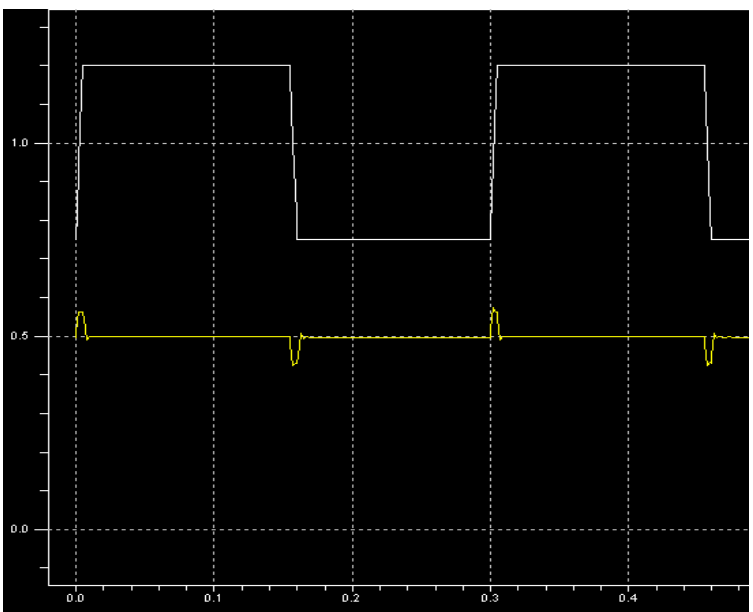

Fig.5 Line regulation of proposed regulator
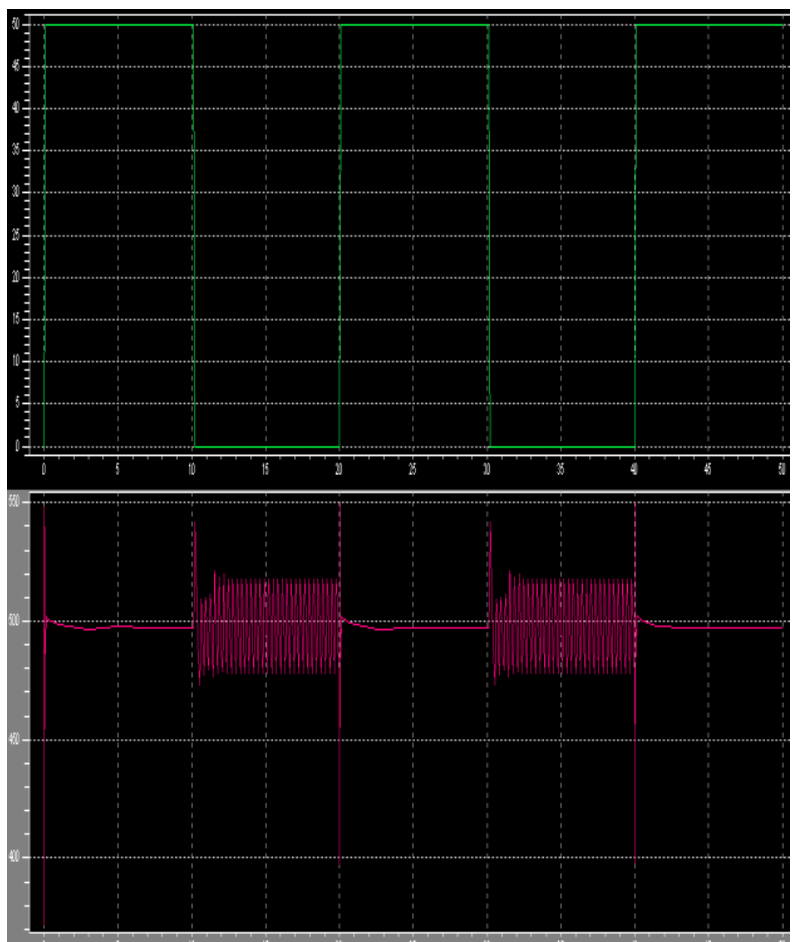

Fig.6 Load Regulation of proposed regulator

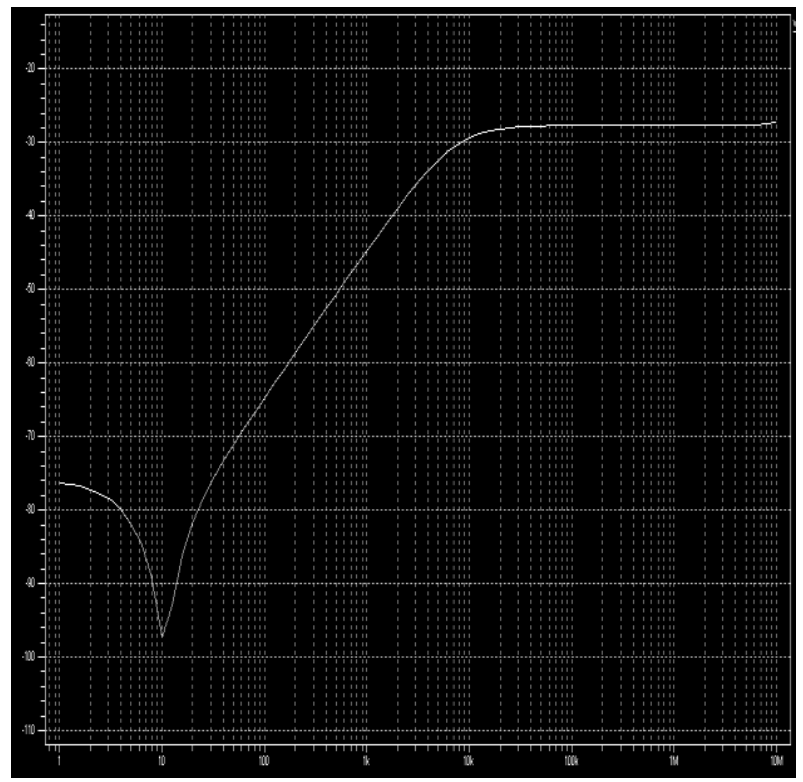

Fig 7: PSRR of proposed regulator

Table 1

\begin{tabular}{|l|l|l|}
\hline Parameters & Base & Modified \\
\hline $\mathrm{V}_{\text {IN }}$ & $.75-1.2 \mathrm{~V}$ & $.75-1.2 \mathrm{~V}$ \\
\hline $\mathrm{V}_{\text {OUT }}$ & $500 \mathrm{mV}$ & $500 \mathrm{mV}$ \\
\hline Dropout Voltage & $250 \mathrm{mV}$ & $220 \mathrm{mV}$ \\
\hline PSRR & $44.5 \mathrm{db} @ 1 \mathrm{Khz}$ & $45 \mathrm{db} @ 1 \mathrm{Khz}$ \\
\hline I Load ${ }_{\text {MAX }}$ & $50 \mathrm{~mA}$ & $80 \mathrm{~mA}$ \\
\hline $\mathrm{I}_{\mathrm{Q}}$ & $16 \mathrm{uA}$ & $7 \mathrm{uA}$ \\
\hline $\begin{array}{l}\text { Line Regulation } \\
(.75 \mathrm{v}-1.2 \mathrm{~V})\end{array}$ & $+66 \mathrm{mV}$ and $-72 \mathrm{mV}$ & $+63 \mathrm{mV}$ and - \\
& & $70 \mathrm{mV}$ \\
\hline $\begin{array}{l}\text { Load regulation }(0- \\
50 \mathrm{~mA})\end{array}$ & $+165 \mathrm{mV}$ and - & $+50 \mathrm{mV}$ and - \\
\hline
\end{tabular}

The dropout voltage is reduced in proposed circuit from $250 \mathrm{mV}$ to $220 \mathrm{mV}$ by performing DC analysis and regulated to $500 \mathrm{mV}$ at $0.75 \mathrm{~V}-1.2 \mathrm{~V}$. The line regulation is performed by changing input voltage. The input voltage is being varied from $0.75 \mathrm{~V}$ to $1.5 \mathrm{~V}$ and the regulated output voltage is $500 \mathrm{mV}$ by reducing fluctuations from $+66 \mathrm{mV}$ and $-72 \mathrm{mV}$ to $+63 \mathrm{mV}$ and $-70 \mathrm{mV}$.

In proposed regulator, the maximum load current is also increased from $50 \mathrm{~mA}$ to $80 \mathrm{~mA}$ and at $80 \mathrm{~mA}$ load current, the output regulated voltage is $500 \mathrm{mV}$ reducing the fluctuations from $+165 \mathrm{mV}$ and $-331 \mathrm{mV}$ to $+50 \mathrm{mV}$ and $-102 \mathrm{mV}$ at $50 \mathrm{~mA}$ comparing with conventional regulator. The Power supply rejection ratio is also increased from $44.5 \mathrm{db} @ 1 \mathrm{Khz}$ to $45 \mathrm{db}$ @ $1 \mathrm{Khz}$ in proposed regulator by adding ac noise of $40 \mathrm{mV}$ with dc voltage at a load current of $50 \mathrm{~mA}$ comparing with conventional regulator.

The quiescent current is also reduced from $16 \mathrm{uA}$ to $7 \mathrm{uA}$ at input voltage of $1.2 \mathrm{~V}$ and dc load current of $50 \mathrm{~mA}$ 
comparing with conventional regulator and thus the power consumption has been reduced across the components of regulator.

\section{CONCLUSION}

With the modified push-pull composite power transistor, quiescent current is lessened significantly and also the high impedance node at the output of error amplifier and the parasitic capacitance at the gate of the power-transistor are decoupled. This improves the stability performance as revealed by stability analysis.

\section{REFERENCES}

[1] Y.-H. Lee, Y.-Y. Yang, K.-H. Chen, Y.-H. Lin, S.-J. Wang, K.-L. Zheng, P.-F. Chen, C.-Y. Hsieh, Y.-Z. Ke, Y.-K. Chen, and C.-C. Huang, "A DVS embedded system power management for high efficiency integrated SoC in UWB system," IEEE J. Solid-State Circuits, vol. 45, no. 11, pp. 2227-2238, Nov. 2010.

[2] M. El-Nozahi, A. Amer, J. Torres, K. Entesari, and E. Sanchez-Sinencio, "High PSR low drop-out regulator with feed-forward ripple cancellation technique," IEEE J. Solid-State Circuits, vol. 45, no. 3, pp. 565-577, Mar. 2010 .

[3] G. A. Rincon-Mora and P. E. Allen, "A low-voltage, low quiescent current, low drop-out regulator," IEEE J. Solid-State Circuits, vol. 33, no. 1, pp. 36-44, Jan. 1998.
[4] . Guo and K. N. Leung, "A 6- $\mu \mathrm{W}$ chip-area-efficient outputcapacitorless LDO in 90-nm CMOS technology," IEEE J. Solid-State Circuits, vol. 45, no. 9, pp. 18961905, Sep. 2010.

[5] T. Y. Man, P. K. T. Mok, and M. Chan, "A high slewrate push-pull output amplifier for low-quiescent current low-dropout regulators with transient-response improvement," IEEE Trans. Circuits Syst. II, Exp. Briefs, vol. 54, no. 9, pp. 755-759, Sep. 2007.

[6] C.-M. Chen, T.-W. Hung, and C.-C. Hung, "Fast transient low-dropout voltage regulator with hybrid dynamic biasing technique for SoC application," IEEE Trans. Very Large Scale Integr. (VLSI) Syst., vol. 21, no. 9, pp. 1742-1747, Sep. 2013.

[7] P. Hazucha, T. Karnik, B. A. Bloechel, C. Parsons, D. Finan, and S. Borkar, "Area-efficient linear regulator with ultra-fast load regulation", IEEE J. Solid-State Circuits, vol. 40, no. 4, pp. 933-940, Apr. 2005.

[8] P. Y. Or and K. N. Leung, "An output-capacitor less low-dropout regulator with direct voltage-spike detection," IEEE J. Solid-State Circuits, vol. 45, no. 2, pp. 458-466, Feb. 2010.

[9] C. M. Chen and C. C. Hung, "A fast self-reacting capacitor-less low-dropout regulator," in Proc. ESSCIRC, Sep. 2011, pp. 375-378. 